hep-ph/9507345

HUTP-95/A025

\title{
Pseudoscalar Conversion and X-rays from the Sun
}

\author{
Eric D. Carlson* \\ Lyman Laboratory of Physics, Harvard University \\ Cambridge, Massachusetts 02138 \\ Li-Sheng Tseng \\ Lyman Laboratory of Physics, Harvard University \\ Cambridge, Massachusetts 02138
}

(July 18, 1995)

\begin{abstract}
We investigate the detection of a pseudoscalar $\phi$ that couples electromagnetically via an interaction $\frac{1}{4} g \phi F \tilde{F}$. In particular, we focus on the conversion of pseudoscalars produced in the sun's interior in the presence of the sun's external magnetic dipole field and sunspotrelated magnetic fields. We find that the sunspot approach is superior. Measurements by the SXT on the Yohkoh satellite can measure the coupling constant down to $g=0.5-1 \times$ $10^{-10} \mathrm{GeV}^{-1}$, provided the pseudoscalar mass $m<7 \times 10^{-6} \mathrm{eV}$, which makes it competitive with other astrophysical approaches.
\end{abstract}

${ }^{*}$ Address after August 1: Olin Physical Laboratory, Wake Forest University, Winston-Salem NC 27109 


\section{INTRODUCTION}

Light neutral pseudoscalars arise naturally as a result of spontaneously broken global symmetries [1]]. They will be truly massless if the symmetry is not anomalous. Although some models can be directly tested in colliders, some of the best limits come from astrophysics.

Such particles generically couple to the electromagnetic field through couplings of the form

$$
\mathcal{L}=\frac{1}{4} g \phi F^{\mu \nu} \tilde{F}_{\mu \nu}
$$

where $\tilde{F}_{\mu \nu}$ is the dual of the electromagnetic field $F^{\mu \nu}$ and $g$ is a coupling constant. This coupling can lead to the interconversion of photons and pseudoscalars in a background electric or magnetic field.

It is reasonable to attempt to use such a coupling to attempt to detect or exclude pseudoscalars of a given mass or coupling. There has been a recent resurgence of interest in detection using such couplings. Several astrophysical approaches have been considered for detecting these electromagnetic couplings [2], including penetration of light from distant stars through intervening opaque clouds [3], the effect on evolution of stars [4, [4], and the appearance of X-rays generated in the cores of distant stars that have been double converted to pseudoscalars and back to photons again [6]. In all but the first of these methods, the pseudoscalars are generated from thermal photons in the presence of nuclear electric fields.

The sun is the nearest and hence relatively brightest source of pseudoscalars. Naturally it has been the source of some recent pseudoscalars search proposals and experiments. Lazarus and his collaborators searched for solar pseudoscalars by attempting to detect a signal from pseudoscalar conversion in a static magnetic field on the earth [7]. The probability of pseudoscalar conversion in such a helioscope is proportional to $g^{2} B^{2} \ell^{2}$. For this experiment, where the magnetic field is $B=2.2 \times 10^{4}$ gauss and its length $\ell=1.8 \mathrm{~m}$, failure to detect a signal set the limit at $g=3.6 \times 10^{-9} \mathrm{GeV}^{-1}$, provided the pseudoscalar mass $m<0.03 \mathrm{eV}$. A 
more expansive search is taking place in Novosibirsk, where a gimballed accelerator dipole magnet tracks the sun $[8]$. With $B=6 \times 10^{4}$ gauss and $\ell=6 \mathrm{~m}$, the detector should be able to provide sensitivity down to about $g=10^{-10} \mathrm{GeV}^{-1}$, provided $m<0.1 \mathrm{eV}$. Given that maintaining a much stronger and larger magnetic field than the one used in Novosibirsk may not be possible, the limit from the Novosibirsk search may be the best attainable from this method of pseudoscalar-photon conversion on earth.

There are, however, other sources of magnetic field in which solar pseudoscalars can convert to photons. On the surface of the sun, there exist many highly dynamic magnetic structures. Pseudoscalars emerging from the sun's interior may convert to X-rays in the sun's own external magnetic fields. These X-rays should be detectable by solar X-ray telescope, such as the SXT on the Yohkoh satellite.

Two strong candidates for the source of pseudoscalar conversion in the sun's exterior are the dipole field and sunspot-related fields. The sun's dipole field, although rather weak, has an effective length of a few hundred thousand kilometers. Sunspots have field strengths of a few kilogauss on the sun's surface with an effective length of no less than a few thousand kilometers. We find that the magnetic field of the sunspot produces a significantly larger X-ray count rate than the sun's general dipole field. Furthermore, using the magnetic field of a large sunspot, it is possible with Yohkoh's SXT to set the limit $g<0.5-1 \times 10^{-10} \mathrm{GeV}^{-1}$, provided $m<7 \times 10^{-6} \mathrm{eV}$ if no X-ray signature from pseudoscalar conversion is detected. This makes this method competitive with other approaches. However, the region we are able to exclude is almost completely covered by the horizontal branch star approach, which covers a higher mass range and couplings down to $6 \times 10^{-11} \mathrm{GeV}^{-1}$. Because of the importance of redundant checking in astrophysics, and the relative ease of completing this analysis, we feel this approach is worthy of further consideration.

In section 2 we discuss the production of pseudoscalars from the sun's interior. In section 3 we discuss their conversion to photons in the presence of the sun's external magnetic fields and the limit which such conversion can achieve. Detection of converted photons by Yohkoh's SXT is discussed in section 4 . We summarize our findings in section 5. 


\section{PRODUCTION OF PSEUDOSCALARS IN THE SUN}

Pseudoscalars with a coupling of the form (1.1) are produced primarily by the Primakoff process. In vacuum the cross section for this process is logarithmically infinite, due to the infinite range of the Coulomb potential. However, at finite temperature and in a dense medium such as the sun's, Debye screening cuts off this divergence and leads to a conversion rate per unit time for photon to pseudoscalar of [9]

$$
\Gamma=\frac{g^{2} \kappa^{2} T}{32 \pi}\left[\left(1+\frac{\kappa^{2}}{4 E^{2}}\right) \ln \left(1+\frac{4 E^{2}}{\kappa^{2}}\right)-1\right],
$$

where $E$ is the energy of a photon, $T$ is the temperature, and $\kappa$ is the inverse Debye screening length. Noting that all except heavy elements, which constitute less than $1 \%$ of the total mass fraction of sun's interior, dissociate completely, we shall assume for simplicity the complete dissociation of all elements in the sun which gives

$$
\kappa^{2}=\frac{4 \pi \alpha}{T} \sum_{i} n_{i}\left(Z_{i}^{2}+Z_{i}\right)
$$

where $n_{i}$ and $Z_{i}$ are the number density and charge of each component of the local density, respectively, and $\alpha$ is the fine structure constant. Multiplying (2.1) times the density of thermal photons, we find the pseudoscalar production density per unit energy per second is given by

$$
\frac{d \Gamma}{d V d E}=\frac{g^{2} \xi^{2} T^{3}}{8 \pi^{3}\left(e^{E / T}-1\right)}\left[\left(E^{2}+\xi^{2} T^{2}\right) \ln \left(1+E^{2} / \xi^{2} T^{2}\right)-E^{2}\right]
$$

where $\xi^{2}=\kappa^{2} / 4 T^{2}$.

We now integrate this over a solar model, for which we use the model of Bahcall and Ulrich [10]. The values of $T$ and $\xi^{2}$ as functions of radius are graphed in Fig. 1. The nearness of the sun enables us to distinguish pseudoscalars emerging from different points on the sun's disk. This suggests that we should not integrate pseudoscalar production throughout the volume of the sun, but only along a line of sight. Since the earth-sun distance is much greater than the sun's radius, we shall treat the lines of sight as parallel. The total rate of production along a line of sight is thus given by 


$$
\frac{d \Gamma}{d x d y d E}=\frac{g^{2} R_{\odot}}{4 \pi^{3}} F(E, b)
$$

where the function $F(E, b)$ is defined as

$$
F(E, b)=\int_{0}^{\sqrt{1-b^{2}}} d z\left\{\frac{\xi^{2} T^{3}}{\left(e^{E / T}-1\right)}\left[\left(E^{2}+\xi^{2} T^{2}\right) \ln \left(1+E^{2} / \xi^{2} T^{2}\right)-E^{2}\right]\right\}_{r=R_{\odot} \sqrt{z^{2}+b^{2}}}
$$

where $\xi^{2}$ and $T$ are implicit functions of $r$, the distance from the center of the sun, and $b$ is

defined so that $b R_{\odot}$ is the impact parameter between the line of sight and the center of the sun. Values of $F(E, b)$ for different energies at various $b$ are graphed in Fig. 2.

Note that most of the pseudoscalars are produced at $b<0.2$. Because this is small, pseudoscalars emerge almost perpendicular to the sun's surface. In subsequent calculations, we will treat the sun's surface as flat, and the pseudoscalars as emerging directly along a perpendicular. This leads to errors proportional to $b^{2}$, or only a few percent. Once the pseudoscalars emerge from the surface of the sun, they can interact with the sun's exterior magnetic field and back-convert into X-rays. We now proceed to find pseudoscalars' contributions to solar X-rays.

\section{CONVERSION OF PSEUDOSCALARS IN THE SUN'S MAGNETIC FIELD}

In the presence of the sun's exterior magnetic field, pseudoscalars can be converted into photons. The probability of conversion along a line of sight is given by [3]

$$
P=\frac{1}{4} g^{2}|\vec{D}(x, y)|^{2}
$$

where we have introduced $\vec{D}(x, y)$ defined as

$$
\vec{D}(x, y)=\int_{0}^{L} \vec{B}_{\perp}(x, y, z) e^{i \theta(z)} d z
$$

where we have taken the line of sight to be the $z$ direction, $z=0$ being where the pseudoscalars leave the sun, and $z=L$ being the earth. The phase $\theta(z)$ has two sources: the

mass of the pseudoscalar (if any) and the effective plasma mass of the photon due to the presence of plasma outside the sun. In general $\theta(z)$ will be given by 


$$
\theta(z)=\int_{0}^{z}\left(\frac{2 \pi \alpha n_{e}\left(z^{\prime}\right)}{m_{e} E}-\frac{m^{2}}{2 E}\right) d z^{\prime} .
$$

where $m_{e}$ is the mass of the electron and $n_{e}$ is the total number density of electrons, and $m$ is the mass of the pseudoscalar.

We will find later that there is only a significant effect if the pseudoscalar is very light. Let us assume for the moment that the pseudoscalar is massless or so light that its contribution to the phase is negligible. The rapidly falling nature of the sun's coronal density outside the chromosphere assures that the phase will oscillate rapidly close to the sun and not change at all away from the sun. The rapid oscillations will wipe out the contribution from the magnetic fields very near the sun. For simplicity, we assume that the contribution is zero from this nearby zone, and that the phase $\theta(z)$ is constant outside this zone. We define the boundary height $h$ by the somewhat arbitrary condition $\frac{\pi}{2}=\theta(\infty)-\theta(h)$. Using numerical models [11] for the sun's chromosphere and corona, we find $h=1200 \mathrm{~km}$ above the "surface" of the sun, defined as the one optical depth level of the sun at a wavelength of $500 \mathrm{~nm}$. We can replace the upper limit on integration by $\infty$, because of the short range of the solar magnetic fields, so our formula for $\vec{D}$ is modified to

$$
\vec{D}(x, y)=\int_{h}^{\infty} \vec{B}_{\perp}(x, y, z) d z
$$

We then integrate the production rate (2.4) and conversion probability (3.1) over the regions of interest on the sun's surface and divide by the solid angle of the sun to the earth to give

$$
\frac{d \Phi}{d E}=\frac{1}{4 \pi L^{2}} \int d x \int d y \frac{d \Gamma}{d E d x d y} P=\frac{g^{4} R_{\odot}}{64 \pi^{4} L^{2}} \int d x d y F(E, b)|\vec{D}(x, y)|^{2}
$$

the differential X-ray flux at earth due to solar pseudoscalar-photon coupling, where $b$ is an implicit function of $x$ and $y$. This general equation will be used to calculate the expected X-ray count rate coming from two components of sun's exterior magnetic field; namely, the general dipole field, and sunspots.

The sun's general field is most apparent during the periods of sunspot minimum. During these periods, magnetographs measure a weak field of about one gauss around the poles 
[12]. Evidence that these polar fields have opposite polarities have led to the belief that this general field is a dipole. Although the solar dynamo model predicts the 22-year cycle of the solar dipole moment [13], the exact behavior of the general field is still uncertain. For example, in 1957, just two years after the sunspot minimum, it was detected that the two poles had the same polarity for a period of 18 months. Nevertheless, we shall assume the existence of the solar dipole field and calculate its contribution to the X-ray count rate during periods of sunspot minimum.

The height $h$ where we can neglect plasma effects is small compared to the scale of the dipole field, so to find $\vec{D}$ we begin integration at the sun's surface. Since the magnetic field of a dipole at the equator is half the strength at the pole, and a dipole field falls as the cube of the distance, we can find the value of $\vec{D}$ along our line of sight to the center of the sun as $\left|\vec{D}_{c}\right|=\frac{1}{4} B_{p} R_{\odot}$, where $B_{p}$ is the magnetic field at the pole. We have explicitly calculated $|\vec{D}(x, y)|^{2}$ as a function of $x$ and $y$, and we find there is very small variation over the region $b<0.2$ where pseudoscalar production is important. So we can approximate $|\vec{D}(x, y)|=\left|\vec{D}_{c}\right|$ everywhere, and hence obtain with the help of (3.5)

$$
\frac{d \Phi_{\text {dip }}}{d E}=\frac{g^{4} R_{\odot}^{5} B_{p}^{2}}{512 \pi^{3} L^{2}} \int_{0}^{1} b F(E, b) d b .
$$

Letting $g=10^{-10} \mathrm{GeV}^{-1}$ and $B_{p}=1$ gauss, values of (3.6) for different energies are graphed in Fig. 3. As evident from Fig. 3, the sun's dipole field as the source of the magnetic field for pseudoscalar conversion does not give a significant X-ray count rate. The signal would be detectable if there were no background, but this, sadly, is not the case. Fortunately, sunspots are a much more promising source, and we now consider them.

As implied by (3.1), strong magnetic field leads to a large pseudoscalar to photon conversion probability. On the sun's surface, the strongest magnetic fields often arise from sunspots. Beside having strong fields, typically several kilogauss, other advantages of sunspots are that they are visible on the sun's disk and that the X-ray background from their central dark region, the umbra, and their surrounding less dark region, the penumbra, is relatively small [14]. Although the shapes of sunspots are often highly irregular, it is convenient to model 
sunspots as having a circular umbra surrounded by an annular penumbra. An approximate field distribution for this model is given by a gaussian [15],

$$
B_{z}(r)=\frac{\phi}{\pi \sigma^{2}} \exp \left(-r^{2} / \sigma^{2}\right)
$$

where $B_{z}$ is the component vertical to the solar surface, $\phi$ is the total magnetic flux coming out of the sunspot, $r$ is the radial distance from the sunspot center, and $\sigma$ is the characteristic size of the sunspot. The radius of the penumbra $p$ is typically about $p^{2}=2.1 \sigma^{2}$. Equation (3.7) is the field roughly at $z=0$. For the sunspots we are interested in, the size of the sunspot will be much greater than $h=1200 \mathrm{~km}$ where we can neglect plasma effects on the X-rays. Presumably, the fields change relatively little over this distance, and we will once again approximate $h=0$.

We now need to know the magnetic field, or, more to the point, $\vec{D}(x, y)$ due to the sunspot. Because the magnetic field is divergenceless, and there is no magnetic field at infinity, we can show

$$
\nabla \cdot \vec{D}(x, y)=B_{z}(x, y, z=0)
$$

If we assume the magnetic field is rotationally symmetric above the surface of the sun, this is sufficient to relate $\vec{D}(r)$ to the magnetic flux $\phi(r)$ within a radius $r$, specifically

$$
\vec{D}(r)=\frac{\phi(r) \hat{r}}{2 \pi r}=\frac{\phi\left[1-\exp \left(-r^{2} / \sigma^{2}\right)\right] \hat{r}}{2 \pi r} .
$$

It can be shown that if the assumption of rotational symmetry is dropped, this formula leads only to an underestimate of the effect.

We now would like to calculate the total X-ray flux from a sunspot. The largest sunspots we will imagine will have a penumbra of size $p=.063 R_{\odot}$ [16. This is somewhat smaller than the scale on which our underlying pseudoscalar production changes. It therefore seems not unreasonable to approximate $F(E, b)$ in $(3.5)$ by the value at the center of the sunspot. Numerical calculations approximating the variation of $F(E, b)$ throughout a very large sunspot show that this introduces at most a few percent error on the X-ray count rate. For a more 
typical sunspot, $p=.02 R_{\odot}$, the error drops below $1 \%$. With these two simplifications and (3.5), we find that the differential X-ray flux for sunspots is given by

$$
\frac{d \Phi_{s}}{d E}=\frac{g^{4} R_{\odot} F(E, b)}{64 \pi^{4} L^{2}} \int_{0}^{p} 2 \pi r d r|\vec{D}(r)|^{2}=\frac{g^{4} R_{\odot} \phi^{2} F(E, b)}{128 \pi^{5} L^{2}}[0.35]
$$

Letting $g=10^{-10} \mathrm{GeV}^{-1}$ and optimistically setting $\phi=10^{23}$ maxwells, which implies a very large sunspot with $p \approx .06 R_{\odot}$ and $B_{z}(0) \approx 3900$ gauss, we find

$$
\frac{d \Phi_{s}}{d E}=540\left(\frac{F(E, b)}{\mathrm{keV}^{5}}\right) \operatorname{counts~s}^{-1} \mathrm{~cm}^{-2} \mathrm{keV}^{-1} .
$$

The maximum value at $E=4 \mathrm{keV}$ and $b=0$ gives about 410 counts $\mathrm{cm}^{-2} \mathrm{~s}^{-1} \mathrm{keV}^{-1}$.

Both (3.5) and (3.10) assume that pseudoscalars are massless particles. If they are not massless, the count rate given above can decrease significantly. As seen in (3.3), the conversion of massive pseudoscalars along different points along the line of sight will produce photons of varying phase. For a sunspot with $\phi=10^{23}$ maxwells, we find that pseudoscalars with mass $m<10^{-5} \mathrm{eV}$ and energy $E=4 \mathrm{keV}$ can cause a phase shift no greater than $\pi / 2$; hence, the decrease in count rate would be minimal. However, for masses that are much greater, incoherent contributions of photons make the method proposed here unrealistic. Realistic pseudoscalar masses are either zero or typically in the range of $10^{-5}-10^{-2} \mathrm{eV}$ [5]. For most of this range, the signal is reduced, probably to invisibility. But for masses near $10^{-5} \mathrm{eV}$, there will be substantial signal, though somewhat reduced from the signal calculated in this paper. We will not calculate in the boundary region $m \simeq 10^{-5} \mathrm{eV}$ explicitly, but will simply state that any limits obtained by this method using a detector that sees X-rays around $4 \mathrm{keV}$ will apply only for masses $m<10^{-5} \mathrm{eV}$.

\section{DETECTION OF X-RAYS CONVERTED FROM SOLAR PSEUDOSCALARS}

As evident from Fig. 2, most of the solar X-rays arising from pseudoscalar-photon conversion are in the energy range of $1-15 \mathrm{keV}$. At present, the best detector for finding such $\mathrm{X}$-rays is the Yohkoh satellite, which is equipped with X-ray detecting instruments for the 
primary purpose of studying solar flares. In particular, the Soft X-ray Telescope (SXT) on Yohkoh forms X-ray images in the 0.25 to $4.0 \mathrm{keV}$ range [17]. Because the expected signal detected by the SXT will be concentrated around $3 \mathrm{keV}$, we will only assume validity of our result for masses $m<7 \times 10^{-6} \mathrm{eV}$.

SXT has an effective area in this energy region of a little under $1 \mathrm{~cm}^{2}$. Multiplying SXT's unfiltered effective area with (3.10) and integrating through the energy range, we obtain the count rate detected by SXT from a sunspot due to pseudoscalars. Once again, assuming $g=10^{-10} \mathrm{GeV}^{-1}$ and a very large sunspot such that $\phi=10^{23}$ maxwells, we obtain SXT's expected count rates for various $b$, as graphed in Fig. 4 with the maximum count rate being around 190 counts/s at $b=0$. (In contrast, the signal from the whole quiet sun dipole field will be only 0.04 counts/s.)

Unfortunately, the signal is dominated by background, which for such a large sunspot would be of the order of 4100 counts/s [14]. This does not necessarily make our signal unobservable. A single sunspot could be watched while it traverses across the face of the sun. The rate could be measured before it reaches the center of the sun, and compared to the rate while it is exactly on center. If the coupling $g=10^{-10} \mathrm{GeV}^{-1}$, then there should be a four percent increase in the X-ray count rate. If systematic errors can be reduced below this level, then couplings at this level can be discovered or ruled out.

If there are no systematic errors to worry about, then using a $10^{3}$ second exposure would allow one to see signals as low as 11 counts/s at the $5 \sigma$ level. Since the count rate scales as the fourth power of the coupling, this allows us to limit or discover the coupling down to $g=5 \times 10^{-11} \mathrm{GeV}^{-1}$. This assumes we can reduce systematic problems to about the $10^{-3}$ level.

Of course, there are systematic contributions. The penumbra may contain hot spots which produce many more X-rays than the rest of the sunspot. These hot spots may be transient, so that they might, for example, turn on coincidentally as the sunspot crosses the center. This effect can be limited by using the image to identify, isolate, and eliminate these hotspots. 
Perhaps a more difficult problem is that the viewing angle of the sunspot changes as it crosses the face of the sun. This changes the apparent size of the sunspot, as well as changing the thickness of solar atmosphere through which it is viewed. One can argue that such effects should be proportional to $b^{2}$, the square of the impact parameter, and hence should be of the order of only a few percent or so as the sunspot moves from $b=0.2$ to $b=0$. Thus it does not necessarily hamper us at the level of $g=10^{-10} \mathrm{GeV}^{-1}$, and by correcting for it we can probably improve on this limit.

There are a variety of other potential systematic headaches, such as angle-dependent detector efficiencies, evolution of sunspot as it moves, and perhaps others. None of these seem to be insurmountable, but they must all be systematically addressed before the value of $g$ can be reliably discovered or ruled out. We believe that the ultimate limit from this approach using the Yohkoh detector will be in the range $g=0.5-1 \times 10^{-10} \mathrm{GeV}^{-1}$. This is the limit for mass $m<7 \times 10^{-6} \mathrm{eV}$.

\section{CONCLUSIONS}

We have found that searching for X-rays from the sun could limit the photon coupling of a light pseudoscalar. The X-ray production from the sun's dipole moment is negligible, but with slightly optimistic assumptions about sunspots, we can obtain a limit using Yohkoh's SXT from the umbral plus penumbral X-ray signal of about

$$
g<0.5-1 \times 10^{-10} \mathrm{GeV}^{-1} \quad \text { for } \quad m<7 \times 10^{-6} \mathrm{eV} .
$$

This limit requires that we study the time evolution of the X-ray production from sunspots as they cross the center of the sun, looking for a brightening in the region $b<0.1$. The exact limit depends to what extent systematic uncertainties can be eliminated. How does this compare with other approaches?

Raffelt and Dearborn have previously placed limits on this coupling at the level $10^{-10} \mathrm{GeV}^{-1}$ by considering the effects of pseudoscalars on the lifetime of horizontal branch 
stars [⿴囗. Naively, our limit looks as good or better than theirs, but only marginally so. In fact, their limit applies to masses as high as $1 \mathrm{keV}$, and hence their limit applies in the all-important axion case, whereas ours does not. Furthermore, Raffelt has recently reexamined the limit from this approach, and concluded that it can limit the coupling down to $6 \times 10^{-11} \mathrm{GeV}^{-1}$ [5], covering nearly all of the range of our detection.

In a previous paper [6], one of us considered what limit had been set or could be set by looking for X-rays from stars, particularly the nearby supergiant $\alpha$-Ori. A limit of $g<2.5 \times 10^{-11} \mathrm{GeV}^{-1}$ was set by reexamining old data, and it was pointed out that this limit could be improved to the level of $10^{-11} \mathrm{GeV}^{-1}$ if a dedicated observation were made. This is better than we believe the solar approach can achieve, but it applies only to masses below about $10^{-10} \mathrm{eV}$.

Combining the other two limits, we see that Yohkoh has a small window of opportunity for this method for masses in the range $10^{-10}-7 \times 10^{-6} \mathrm{eV}$, if the coupling is very close to $5 \times 10^{-11} \mathrm{GeV}^{-1}$. How likely is this value? It is not favored by any model of which these authors are aware. For such a small coupling, eliminating systematic errors is crucial, and any "discovery" of a signal would be met with justifiable skepticism.

We therefore conclude that the approach we have proposed here is of only marginal value for investigating new portions of parameter space. On the other hand, more information is better. In the field of astrophysics, one never knows when some limit will be called into question, and it is always good to have redundant approaches to particle physics limits. Redundant approaches are as close as we can come to repeatability of experiments in astroparticle physics, so we should seize every opportunity we can find. Who knows? Maybe this approach will find evidence for pseudoscalars, or perhaps just lead to a better understanding of X-rays from sunspots. 


\section{ACKNOWLEDGMENTS}

We would like to thank H. Hudson for providing extremely helpful information concerning Yohkoh's SXT. We would also like to thank G. Field for his helpful input to this project. Additional thanks go to J. Bookbinder and L. Golub for helpful comments on solar X-ray detection. This research was supported by the National Science Foundation under contract NSF-PHY-92-18167, and also by a Sloan Foundation Fellowship. L.S. Tseng was supported by a grant from the Harvard University Physics Department Undergraduate Research Fund. 


\section{REFERENCES}

[1] For a review of light pseudoscalars and their couplings, see, for example, J.E. Kim, Phys. Rep. 150, 1 (1987).

[2] For reviews on methods of pseudoscalars searches, see, M.S. Turner, Phys. Rep. 197, 67 (1990); G.G. Raffelt, Phys. Rep. 198, 1 (1990); E. Massó and R. Toldrà, "On a Light Spinless Particle Coupled to Photons", preprint UAB-FT-361, 1995, (archives hep-ph/9503293), to be published in Phys. Rev. D.

[3] E.D. Carlson and W.D. Garretson, Phys. Lett. B 336, 431 (1994).

[4] G.G. Raffelt and D.S.P. Dearborn, Phys. Rev. D 36, 2211 (1987).

[5] G.G. Raffelt, "Axions in Astrophysics and Cosmology", Contribution to the XVth Memorial Workshop, Villars-sur-Ollon, Switzerland, 1995, (archives hep-ph/9502358).

[6] E.D. Carlson, Phys. Lett. B 344, 245 (1995).

[7] D.M. Lazarus et al., it Phys. Rev. Lett. 69, 2333 (1992).

[8] P.V. Vorobyov and I.V. Kolokolov, "Detectors for the Cosmic Axionic Wind", Talk at International Conference on Cosmoparticle Physics, Moscow, Russia, 1994, (archives astro-ph/9501042).

[9] G.G. Raffelt, Phys. Rev. D 33, 897 (1986).

[10] J.N. Bahcall and R.K. Ulrich, Rev. Modern Phys. 60, 297 (1988).

[11] J.E. Vernazza et al., Ap. J. Suppl. 45, 635 (1981); P. Maltby et al., Ap. J. 306, 284 (1986).

[12] E. Tandberg-Hanssen, Solar Activity, (Blaisdell Publishing Company, Waltham, MA, 1967).

[13] H.W. Babcock, Ap. J. 133, 572 (1961). 
[14] H. Hudson, private communication.

[15] V. Bumba, Izv. Crimea Astrophys. Obs. 23, 212 (1960).

[16] C.W. Allen, Astrophysical Quantities, 3rd ed. (The Athlone Press, London, 1973).

[17] For details concerning SXT, see S. Tsuneta et al., Sol. Phys. 136, 37 (1991).

\section{Figure Captions}

Fig. 1. The temperature (solid line) and $\xi^{2}$ (dashed line) as functions of radius $r$ based on the model of Bahcall and Ulrich [10].

Fig. 2. The function $F(E, b)$ for $b$ from $b=0.0$ (top curve) to $b=0.3$ (bottom curve). The solid lines are at intervals of $\Delta b=0.1$, and the dashed lines are at intervals of $\Delta b=0.02$.

Fig. 3. The expected X-ray signal due to pseudoscalar-photon conversion in the sun's general dipole field, taking $g=10^{-10} \mathrm{GeV}^{-1}$ and $B_{p}=1$ gauss.

Fig. 4. The expected X-ray count rate detected by Yohkoh's unfiltered SXT due to pseudoscalar-photon conversion in the magnetic field of a sunspot, taking $g=$ $10^{-10} \mathrm{GeV}^{-1}$ and $\phi=10^{23}$ maxwells. The sunspot's center defines the impact parameter $b R_{\odot}$ 